\title{
Subcutaneous emphysema of the leg after hardware removal and bone allografting for infected non-union of the distal femur
}

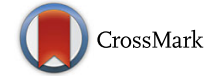

\author{
Vincenzo Denaro ${ }^{1}$, Umile Giuseppe Longo ${ }^{1 *}$, Giuseppe Salvatore ${ }^{1}$, Vincenzo Candela ${ }^{1}$ and Nicola Maffulli, ${ }^{2,3}$
}

\begin{abstract}
Background: Infected non-unions of the supracondylar region of the femur are uncommon. Even though hardware removal is a common procedure, it may lead to complications, including neurovascular injury, refracture, worsening pain or recurrence of deformity.

Case presentation: We report on a male who developed subcutaneous emphysema of the leg after hardware removal and bone allografting for an infected non-union of the distal femur. He was managed by debridement of the surgical wound, antibiotic therapy, multiple fasciotomies, and application of a VAC (vacuum-assisted closure) system.

Conclusions: Although subcutaneous emphysema of the leg after hardware removal and bone allografting for infected non-union of the distal femur is extremely rare, the potential life treating complications and their potential impact on the functional status of the patient have to be taken into consideration when counseling patients about this procedure. Even when it is not possible to identify a bacterial pathogen responsible for the subcutaneous emphysema of the leg, prompt intervention may save the limb of the patient.
\end{abstract}

Keywords: Gangrene, Fracture, Hardware removal, Femur

\section{Background}

Infected non-unions are defined as a state of failure of union and persistence of infection at the fracture site for 6 to 8 months $[1,2]$. Infected non-unions of the supracondylar region of the femur are uncommon [1-3]. However, when they occur, management is challenging. They usually originate after severe open fractures with extensive comminution and segmental bone loss or after internal fixation of a comminuted closed fracture [1, 4]. Factors which complicate management and recovery include osteomyelitis, polybacterial multidrug-resistant infection, osteopenia, soft-tissue loss with multiple sinuses, complex deformities with limb-length inequality, and stiffness of the adjacent joint [5].

Often patients require hardware removal and further orthopaedic interventions. Even though hardware removal is a common procedure, it may lead to complications,

\footnotetext{
* Correspondence: g.longo@unicampus.it

'Department of Orthopaedic and Trauma Surgery, Campus Bio-Medico

University, Via Alvaro del Portillo, 200, 00128 Trigoria, Rome, Italy

Full list of author information is available at the end of the article
}

including neurovascular injury, refracture, worsening pain or recurrence of deformity [6, 7].

We report on a male who developed subcutaneous emphysema of the leg after hardware removal and bone allografting for an infected non-union of the distal femur. He was managed by debridement of the surgical wound, antibiotic therapy, multiple fasciotomies, and application of a VAC (vacuum-assisted closure) system.

To the best of our knowledge, this is the first reported incidence of subcutaneous emphysema of the leg after hardware removal and bone allografting for infected non-union of the distal femur. Our patient was informed that data concerning the case would be submitted for publication, and gave written consent.

\section{Case presentation}

A 59-year-old male sustained an open right supraintercondylar fracture of the distal femur with extensive comminution after an accident car (Fig. 1). After 3 days, the patient underwent an open reduction and internal fixation of the fracture with irrigation and debridement 


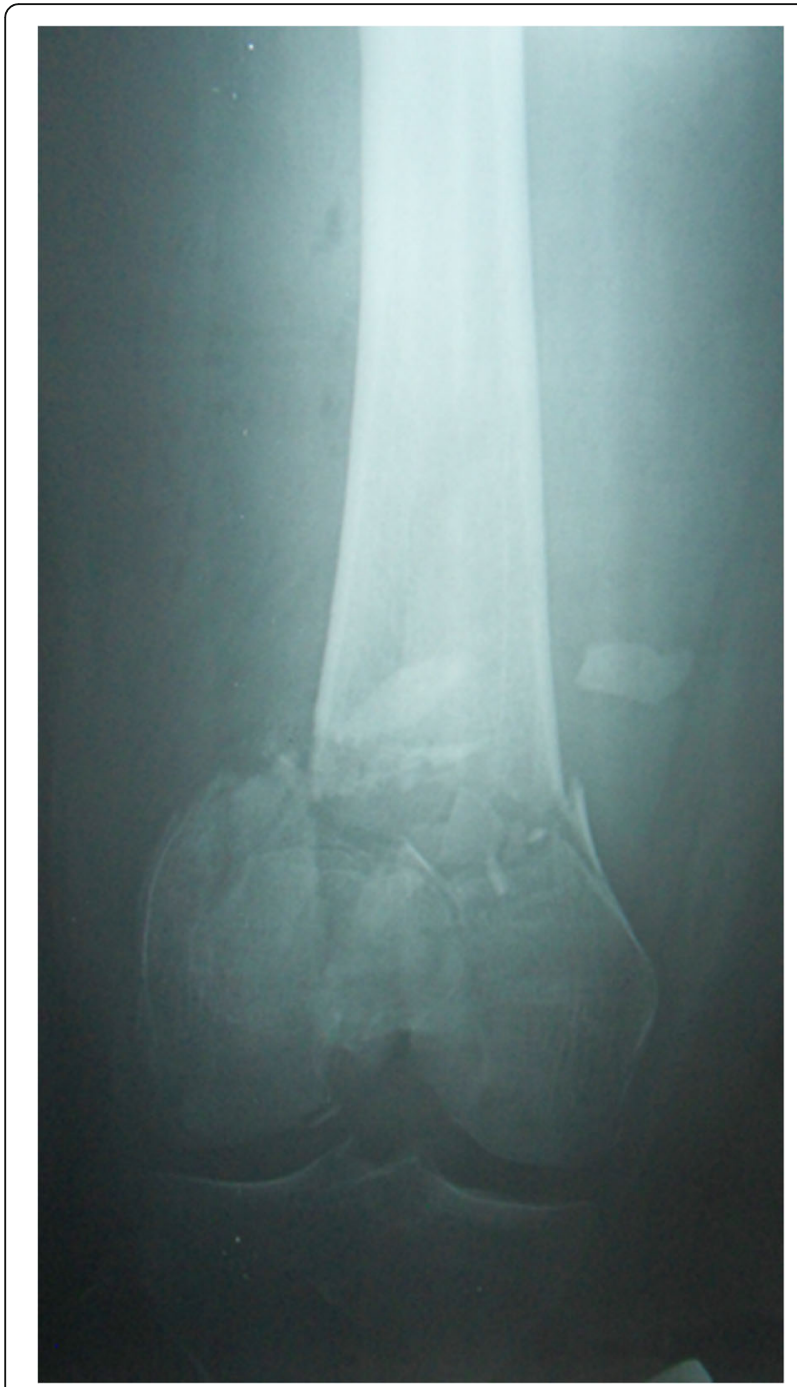

Fig. 1 Anteroposterior radiograph of the right supra-intercondylar multifragmentary fracture of the distal femur

at another hospital (Fig. 2). Post-operatively the patient underwent assisted continuous passive motion and muscular strengthening for 6 months ( $4 \mathrm{~h}$ per day). Weight bearing was allowed 3 months after surgery.

Seven months postoperatively the patient was able to walk with crutches and a knee brace with intensive knee pain. Range of motion of the knee (extension/flexion) was $0-40^{\circ}$. Radiographs of the right knee showed a delayed union of the right supra-intercondylar distal femoral fracture. The patient underwent a knee arthroscopy to perform a release of the adhesions and to gain range of motion. Post-operatively the patient underwent assisted continuous passive motion and muscular strengthening for a 5-month period. Weight bearing was allowed the day after the procedure. Range of motion after 5 months of exhausting physiotherapy was $0-60^{\circ}$ (extension/flexion).

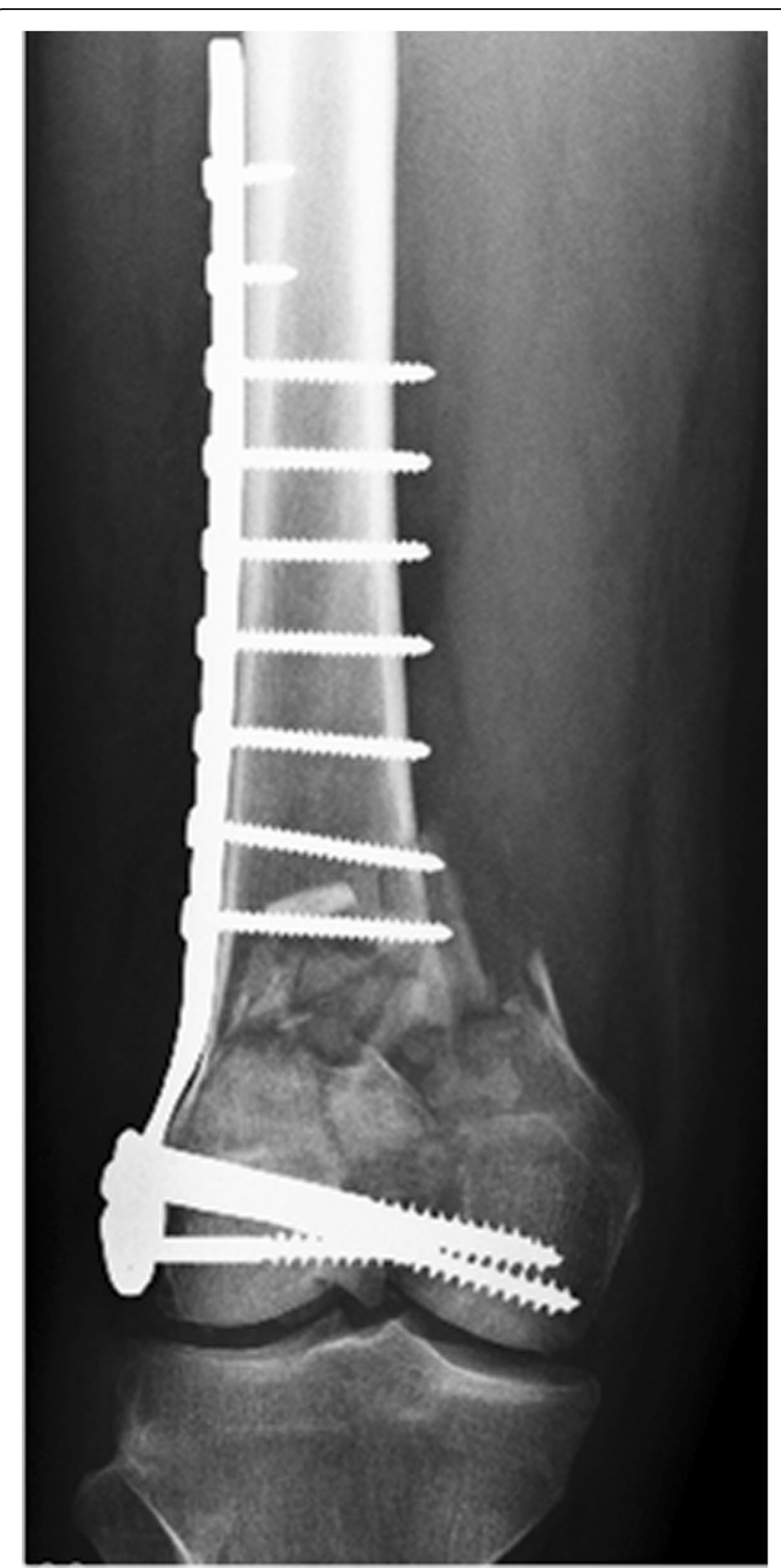

Fig. 2 Anteroposterior radiograph of the right femur 3 days post-operatively

Despite of continuous physiotherapy, at 2 year follow up, the patient was not able to walk without crutches and knee brace, and he had intensive knee pain.

Radiographs of the right knee showed a non complete union of the right supra-intercondylar distal femoral fracture and a varus deformity of the femur (Fig. 3). The patient underwent a labeled leukocyte imaging showing increased uptake in correspondence of the distal femur. Laboratory tests were normal. The infected non-union was classified in type A1 according to Jain AK et al. [5].

The patient underwent hardware removal and bone allograft implantation. Samples for microorganism cultures were obtained at surgery from surgical wound, 


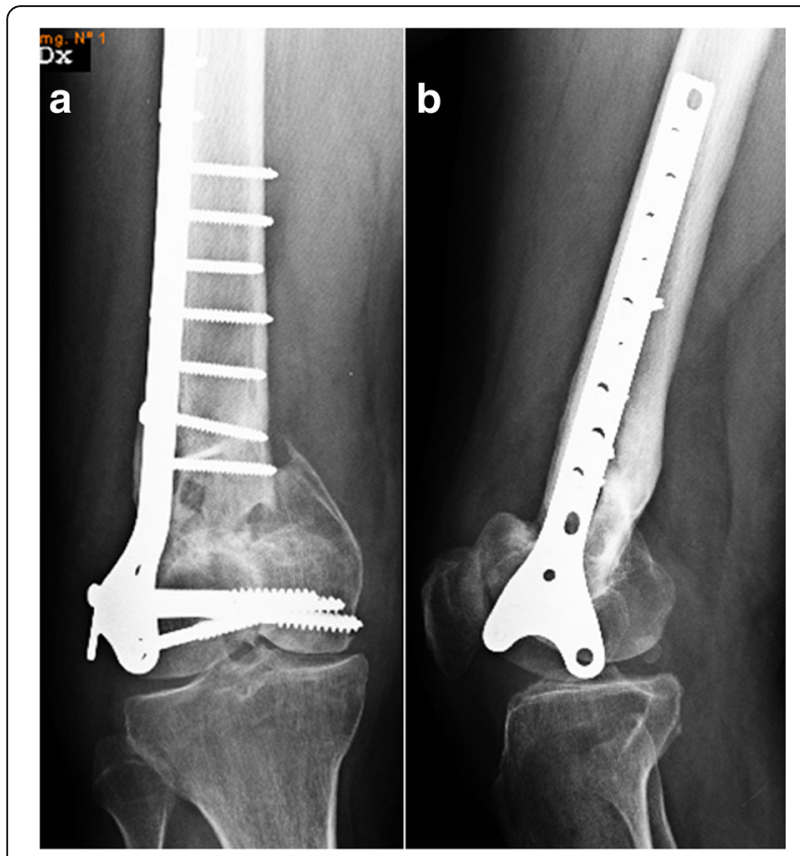

Fig. 3 a Anteroposterior radiograph pre-operative of hardware removal; b Lateral radiograph pre-operative of hardware removal

non-union fibrous tissue, curetted bone and canal marrow cavity, but no pathogens were identified. Culture was performed also on the bone allograft, but no pathogens were identified. After surgery, the patient started antibiotic therapy for the management of the suspected osteomyelitis with teicoplanin $800 \mathrm{mg} /$ die i.v. and levofloxacin $750 \mathrm{mg} /$ die per os.

$24 \mathrm{~h}$ postoperatively, the patient developed emphysema of the right lower limb. A CT scan showed the subcutaneous emphysema of the leg (Fig. 4), with extension to the toes (Fig. 5). The patient underwent exploration and debridement of the surgical wound, and multiple fasciotomies. Samples from surgical wound and soft tissues for microorganism cultures were obtained at surgery, but no pathogens were identified. Postoperatively a VAC (vacuum-assisted closure) system was applied. The patient underwent maximum dosage of broad spectrum antibiotic therapy for 14 days, consisting of ampicillin $18 \mathrm{~g} /$ die i.v., clindamicin $2.4 \mathrm{~g} /$ die i.v. and teicoplanin $800 \mathrm{mg} /$ die i.v. Because increased values of the hepatic enzymes were observed after 3 days, teicoplanin was substituted with vancomycin $2 \mathrm{~g} /$ die i.v.. The patient had complete resolution of subcutaneous emphysema of the leg in the postoperative period. 14 days postoperatively the VAC system was removed, and surgical wounds were closed.

The patient underwent a progressive rehabilitation programme. Weight bearing was allowed since the first day after removal of the VAC. The patient started again

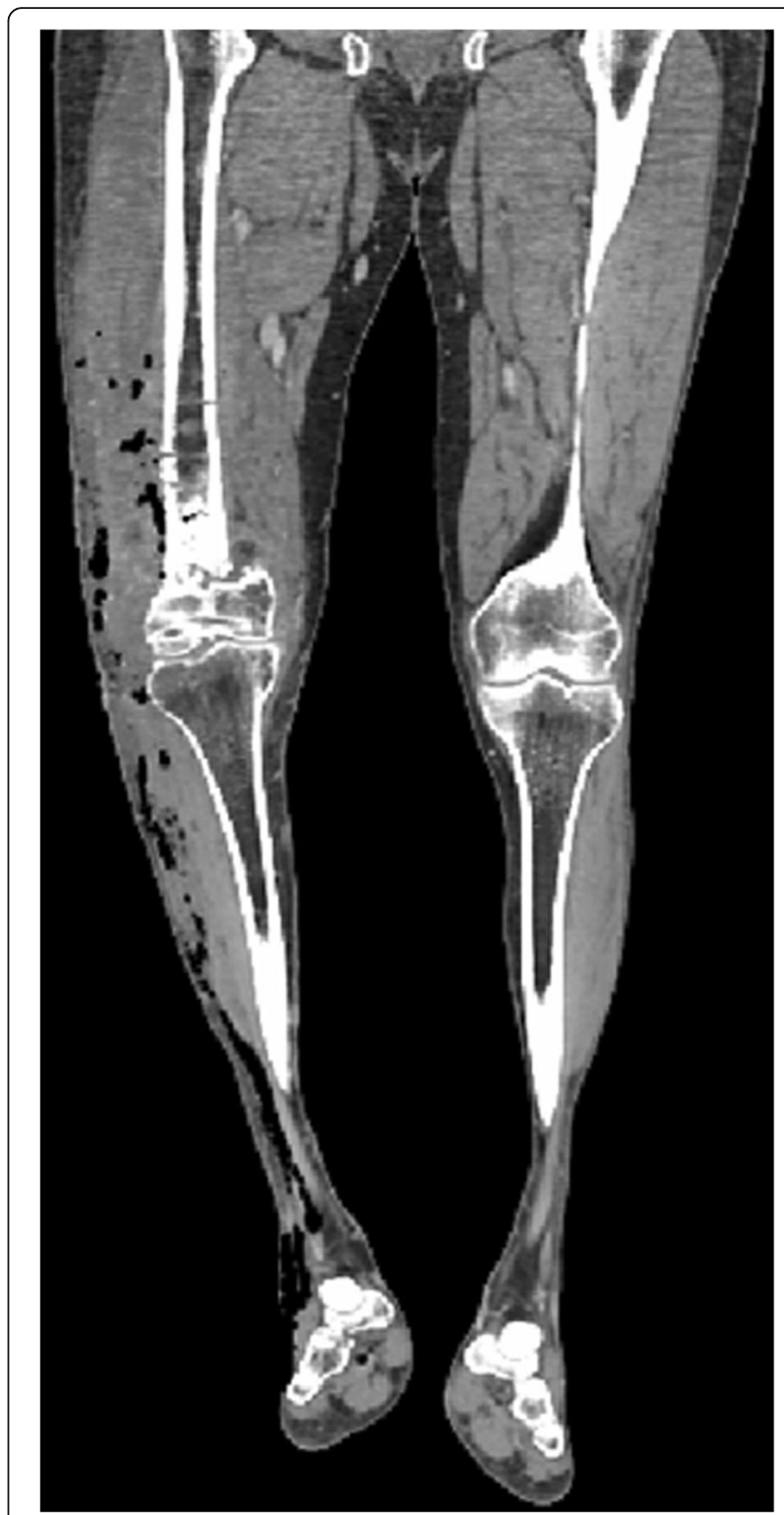

Fig. 4 A CT scan showed the subcutaneous emphysema of the leg

a continuous passive motion for 4 months postoperatively. The range of motion was $0^{\circ}-60^{\circ}$. The patient underwent antibiotic therapy with levofloxacin $500 \mathrm{mg} /$ die and rifampicin $900 \mathrm{mg} /$ die for 3 months.

At 4 year follow-up, the patient underwent radiographs (Fig. 6) and a labeled leukocyte imaging showing absence of uptake in correspondence of the distal femur. The patient's limb was saved.

\section{Discussion}

Hardware removal is a common orthopaedic procedure and it is frequently performed for symptoms attributed to the presence of hardware and in case of implant failure, infection, non-union, and soft-tissue compromise. 


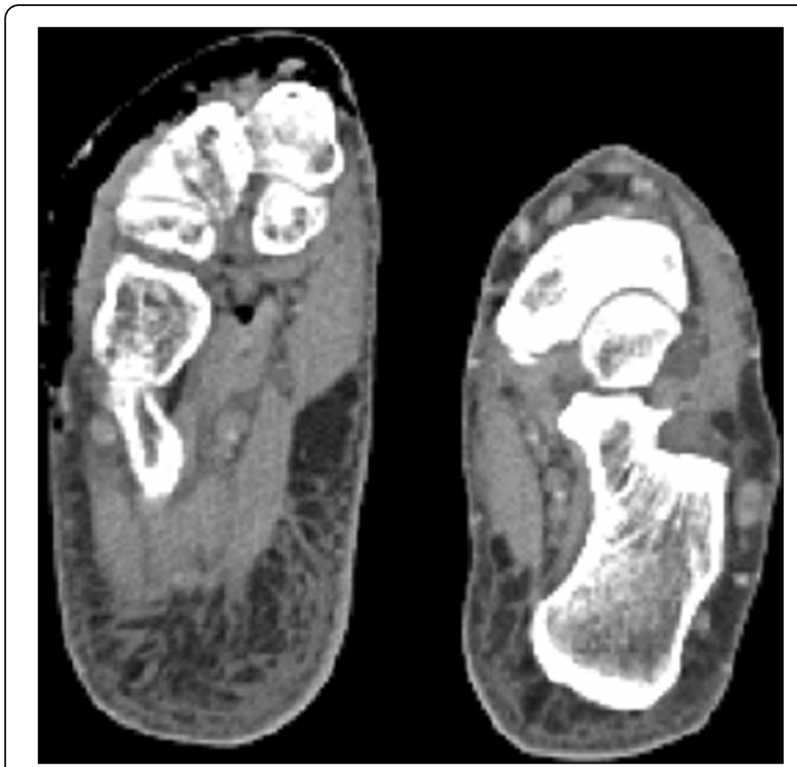

Fig. 5 A CT scan showed extension of the emphysema to the toes

As any surgical procedure, hardware removal carries inherent risks, including wound complications, iatrogenic injury, and anaesthetic complications [8]. The most common complication of hardware removal is infection, although gas gangrene has not been reported to date as a complication of hardware removal $[1,2]$.

Gas gangrene is a life-threatening disease. A traumatic injury, especially outdoor, exposes people to ground or other organic staff that could contain C. perfringens or other bacteria. In this way bacteria are introduced into soft tissues [9]. Healthy oxygenated tissues are hostile environments for the grow of bacteria. However a damage to blood supply suppresses the inflammatory response and allows the uncontrolled colonization of bacteria: the result could be a fulminant gas gangrene. The infection expands to other tissues, so the whole limb could become gangrenous in few hours making the amputation of the limb or removal of all of the infected tissues the only therapeutic strategies. Without this, the toxins produced by the bacteria rapidly kills the patient [9].

Although nonclostridial gas gangrene is not an unusual occurrence, relatively few cases have been reported. Due to the serious nature of some of these infections, it is important for physicians to familiarize themselves with these nonclostridial crepitant infections, which are often confused with clostridial myonecrosis [10]. Unfortunately, despite of our efforts, we were not able to determine the nature of the gangrene (clostridial or non nonclostridial). Multiple samples were performed both at surgery for hardware removal and during fasciotomies, as well as hemoculture and culture of the allograft, but no pathogens were isolated. The results of the cultures were probably a false negative [11]. Otherwise,

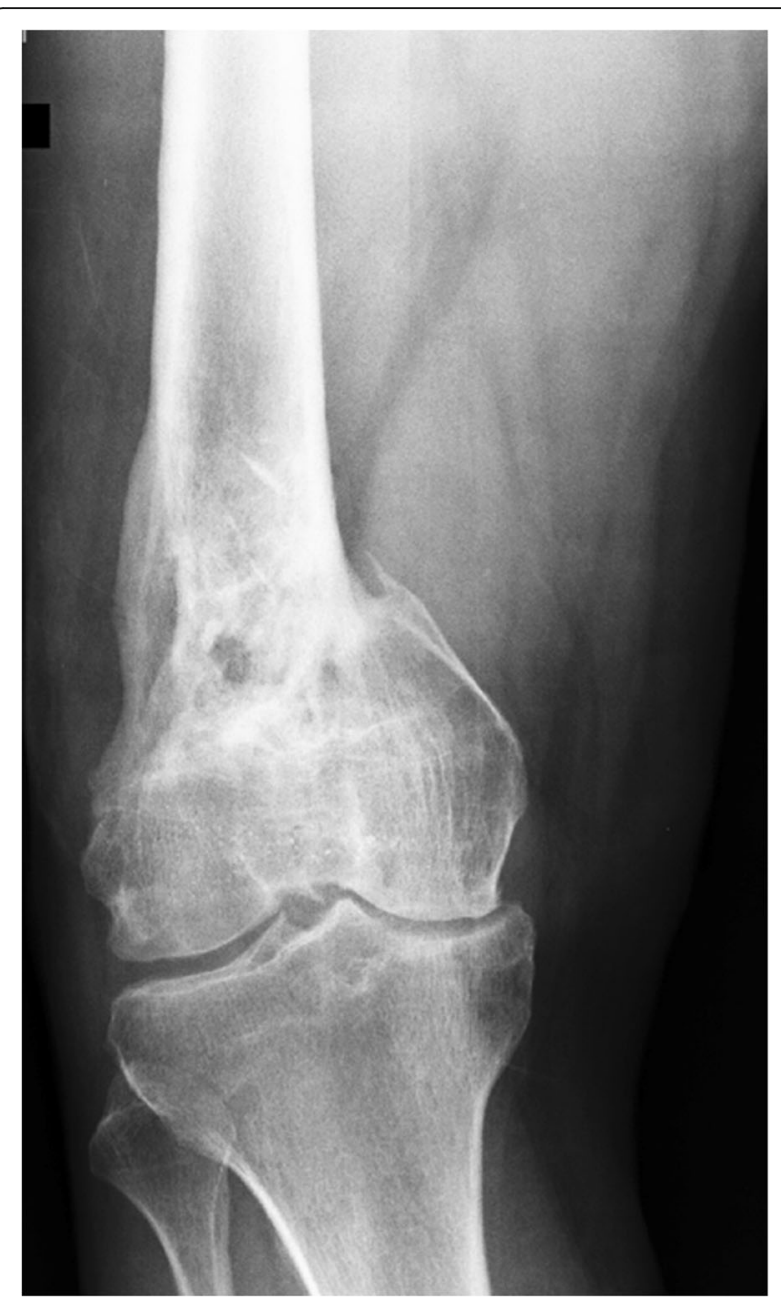

Fig. 6 Anteroposterior radiograph of the right femur 2 year after the subcutaneous emphysema of the leg

we have not a scientific explanation to the emphysema (all the way down to the toes of the foot) and for the increased uptake at the bone scan. In clinical practice, sometimes, despite clinical diagnosis of infection, hemoculture and culture are negative and it is not possible to isolate pathogens responsible of the disease. In these cases, it is important to apply general principal and utilize large spectrum antibiotics. This strategy allowed us to save the limb in this patient.

Primary wound closure remains controversial in these patients, because of concerns for gas gangrene. Partial wound closure is an alternative, with delayed wound closure within 3 to 7 days. The use of sub-atmospheric pressure dressings, available commercially as the VAC device can be a valid alternative option in selected patients [12]. Wounds heal better with a sub-atmospheric pressure of $125 \mathrm{mmHg}$, especially if a pressure cycle of 5 min of suction alternated by 2 min off suction is utilized. In this way the blood flow is optimized, local tissue 
edema decreases, and the excessive liquid from the wound bed is removed. Moreover, the cyclic use of subatmospheric pressure changes the cytoskeleton of the cells in the wound bed and activates a cascade of intracellular signals leading to cell division and formation of granulation tissue. The combination of these mechanisms makes the VAC device a useful instrument for orthopaedic surgeon. Before surgery for hardware removal, we performed a review of the literature, to establish whether to perform a one- or a two- stage procedure: the optimal operative management of infected nonunions was not clear. Union rate for one stage strategies is between 70 and $100 \%$ and persistent infection percentages is between 0 and $55 \%$. Instead for two-stage strategies union rate is between 66 and $100 \%$ and persistent infection percentages is between 0 and $60 \%$. [1]. Because of patient requests and our experience in the field, and because of the low level of evidences (at best level IV, and suggesting similar results), we decided to perform a one-stage procedure with bone allografting. Even allografts are considered sterile, clostridium infections have been described after bone allograft implantation [13-17].

After the death from Clostridium sordellii sepsis of a 23-year-old man who had received a cadaveric musculoskeletal allograft [14], the Centers for Disease Control and Prevention (CDC) monitored the cases of allograftassociated clostridium infections and investigated processing and testing methods used by the tissue bank Recommendations have been made to tissue banks, the American Association of Tissue Banks, and the FDA to reduce the risk of allograft-associated infection to use a validated sporicidal process to confer the greatest protection for patients [14]. The bone allograft we used in our patient underwent the guidelines for allograft preparation. Therefore, we are not able to understand whether the infection arises from the bone allograft or from the site of infected non-union of the right femur of our patient.

\section{Conclusion}

Although subcutaneous emphysema of the leg after hardware removal and bone allografting for infected non-union of the distal femur is extremely rare, the potential life treating complications and their potential impact on the functional status of the patient have to be taken into consideration when counseling patients about this procedure. Even when it is not possible to identify a bacterial pathogen responsible for the subcutaneous emphysema of the leg, prompt intervention may save the limb of the patient.

\section{Abbreviations}

C. perfringens: Clostridium perfringens; CDC: Centers for disease control and prevention; CT: Computed tomography; VAC: Vacuum-assisted closure
Acknowledgements

No one else contributed towards the article except for the authors.

Funding

The authors declare that they have no conflict of interest and no funding source.

Availability of data and materials

All of the data are presented in the manuscript.

Authors' contributions

UGL made substantive intellectual contributions to the published study and wrote the paper, GS and VC evaluated the clinical conditions and the clinical course of the patient during the follow-up period, NM and VD contributed to clinical and instrumental exams interpretations and reviewed the manuscript. All authors read and approved the final manuscript.

\section{Competing interest}

UGL is a member of the Editorial Board of BMC Musculoskeletal Disorders. The remaining authors declare that they have no competing of interest.

Ethics approval and consent to participate Not applicable.

\section{Consent for publication}

The subject allows the publication of the data, A written informed consent was obtained from the patient for publication of this case report and any accompanying images.

\section{Publisher's Note}

Springer Nature remains neutral with regard to jurisdictional claims in published maps and institutional affiliations.

\section{Author details}

'Department of Orthopaedic and Trauma Surgery, Campus Bio-Medico University, Via Alvaro del Portillo, 200, 00128 Trigoria, Rome, Italy. ${ }^{2}$ Centre for Sports and Exercise Medicine, Barts and The London School of Medicine and Dentistry, Mile End Hospital, 275 Bancroft Road, London E1 4DG, UK.

${ }^{3}$ University of Salerno School of Medicine, Salerno, Italy.

Received: 26 March 2017 Accepted: 2 August 2017

Published online: 15 August 2017

References

1. Struijs PA, Poolman RW, Bhandari M. Infected nonunion of the long bones. J Orthop Trauma. 2007;21(7):507-11.

2. Meyer S, Weiland AJ, Willenegger $H$. The treatment of infected non-union of fractures of long bones. Study of sixty-four cases with a five to twenty-oneyear follow-up. J Bone Joint Surg Am. 1975;57(6):836-42.

3. Weng CJ, Wu CC, Feng KF, Tseng IC, Lee PC, Huang YC. Comparison of supraintercondylar and supracondylar femur fractures treated with condylar buttress plates. BMC Musculoskelet Disord. 2016;17(1):413.

4. Chapman MW, Finkemeier CG. Treatment of supracondylar non-unions of the femur with plate fixation and bone graft. J Bone Joint Surg. 1999;81A: 1217-28.

5. Jain AK, Sinha S. Infected nonunion of the long bones. Clin Orthop Relat Res. 2005:431:57-65.

6. Busam ML, Esther RJ, Obremskey WT. Hardware removal: indications and expectations. J Am Acad Orthop Surg. 2006;14(2):113-20.

7. Jain A, Kumar S, Aggarwal AN, Jajodia N. Augmentation of bone healing in delayed and atrophic nonunion of fractures of long bones by partially decalcified bone allograft (decal bone). Indian J Orthop. 2015;49(6):637-42.

8. Bose D, Kugan R, Stubbs D, McNally M. Management of infected nonunion of the long bones by a multidisciplinary team. Bone Joint J. 2015;97-B(6): 814-7.

9. Titball RW. Gas gangrene: an open and closed case. Microbiol (Reading, England). 2005;151(Pt 9):2821-8.

10. Weisenfeld LS, Luzzi A, Picciotti J. Nonclostridial gas gangrene. J Foot Surg. 1990;29(2):141-6.

11. Kainer MA, Strausbaugh $\amalg$, Liedtke LA, Jernigan DB, Archibald LK: IDSA Emerging Infections Network. An overview of allograft-associated infections 
(AAI) in the U.S.- 1998-2002. In: Proceedings of the 40th Annual Infectious Diseases Society of America (IDSA) Meeting, Chicago, October 24-27, 2002: 46. abstract.

12. Venturi ML, Attinger $C E$, Mesbahi AN, Hess CL, Graw KS. Mechanisms and clinical applications of the vacuum-assisted closure (VAC) device: a review. Am J Clin Dermatol. 2005;6(3):185-94.

13. Centers for Disease Control and Prevention (CDC). Unexplained deaths following knee surgery-Minnesota, November 2001. MMWR Morb Mortal Wkly Rep. 2001;50(46):1035-6.

14. Kainer MA, Linden JV, Whaley DN, Holmes HT, Jarvis WR, Jernigan DB, Archibald LK. Clostridium infections associated with musculoskeletal-tissue allografts. N Engl J Med. 2004;350(25):2564-71.

15. Kubosch EJ, Bernstein A, Wolf L, Fretwurst T, Nelson K, Schmal H. Clinical trial and in-vitro study comparing the efficacy of treating bony lesions with allografts versus synthetic or highly-processed xenogeneic bone grafts. BMC Musculoskelet Disord. 2016;17:77.

16. Longo UG, Trovato U, Loppini M, Rizzello G, Khan WS, Maffulli N, Denaro V. Tissue engineered strategies for pseudoarthrosis. Open Orthopaedics J. 2012;6:564-70.

17. Martinez de Albornoz P, Khanna A, Longo UG, Forriol F, Maffulli N. The evidence of low-intensity pulsed ultrasound for in vitro, animal and human fracture healing. Br Med Bull. 2011:100:39-57.

\section{Submit your next manuscript to BioMed Central} and we will help you at every step:

- We accept pre-submission inquiries

- Our selector tool helps you to find the most relevant journal

- We provide round the clock customer support

- Convenient online submission

- Thorough peer review

- Inclusion in PubMed and all major indexing services

- Maximum visibility for your research

Submit your manuscript at www.biomedcentral.com/submit 